\title{
The structure of water-layer sensitivity kernels in OBN-type acquisition geometries
}

Francisco Carlos Lajús Junior, Antonio Henrique da Fontoura Klein Universidade Federal de Santa Catarina - UFSC

Copyright 2021, SBGf - Sociedade Brasileira de Geofísica.

This paper was prepared for presentation at the $17^{\text {th }}$ International Congress of the Brazilian Geophysical Society, held in Rio de Janeiro, Brazil, 8 November to 11 November, 2021.

Contents of this paper are to be reviewed by the Technical Committee of the $17^{\text {th }}$ International Congress of The Brazilian Geophysical Society and do not necessarily represent any position of the SBGf, its officers or members. Electronic reproduction or storage of any part of this paper for commercial purposes without the written consent of The Brazilian Geophysical Society is prohibited.

\section{Abstract}

The water column is usually taken as an homogeneous velocity layer in OBN-type marine seismic surveys. Heterogeneities caused by salinity and temperature variations, however, may introduce significant artifacts resulting in inaccurate interpretation of sub-sea floor geologic features, especially accounting the long time spans of seismic acquisitions that take place in ultra-deep ocean environments. Here we investigate the underlying structure of sensitivity kernels in this horizontally distributed acquisition geometry, mostly focused on the development of improved water layer velocity reconstructions from bottom node data. Two possible formulations are here considered, relating first arrivals with velocity perturbations, where a reference oceanic state and some overall shape/pattern of the velocity perturbations could be known a priori. The results are expected to provide guidelines for adapted inversion schemes, directed to more detailed water layer velocity reconstruction.

\section{Introduction}

In a 3D/4D marine seismic survey, it is quite usual that the acquisition time along sail-lines and between adjacent ones may take several days, weeks, or even months (RITTER, 2010). At the same time, water properties can vary relatively rapidly, both temporally and spatially, as a result of several oceanic phenomena, such as the interaction between warm/cold water fronts, eddies, internal waves, plumes, and currents, to name a few. Particularly in deep water areas, if variations of water properties are not properly accounted during the seismic processing, lateral discontinuities (or jitter) on crossline sections can lead to stack deterioration and migration artifacts (WOMBELL, 1996; BERTRAND et al., 2004; LACOMBE et al., 2009; GUERRA et al., 2015; AYOBAMI et al., 2020).

For streamer data, a number of methods have been already formulated, ranging from straightforward static corrections (WOMBELL, 1996) and NMO adjustments of traveltimes (LACOMBE et al., 2009), to more detailed characterizations obtained from reflections occurring in the water column (BRIGHT et al., 2015). These reflection events are related to impedance contrasts produced by termohaline variations of the water properties, which in turn allows detailed visualization of some oceanic processes
(HOLBROOK et al., 2003; SONG et al., 2012). Such application of classical seismic processing, adapted to emphasize water reflections towards the study of ocean behavior became a research field by itself - known today as Seismic Oceanography (SHEEN, 2019).

Similarly, corrections of water velocity are also necessary in OBN surveys, with some additional care to shot and receiver positions, and timing (UDENGAARD and CRAFT, 2012). Here, joint inversion seems to be recommended, to avoid error leakage to each separated variable (AMINI et al., 2016). Nonetheless, the usual treatment of water layer velocity appears to also assume a homogeneously (spatially-averaged) time-dependent quantities (BOELLE et al., 2010; DOCHERTY and HAYS, 2012; AHMED et al., 2013; AMINI et al., 2016), though further development considers time-dependent corrections of an overall (interpolated) 1D sound velocity profile (DOCHERTY and HAYS, 2012). Alternatives to even more detailed velocity reconstructions involve some additional input, as the information of multiples (reverberation) trapped in the water column (DUNN, 2015), or simultaneous data from vertical cables (ZOU et al., 2018).

In this study we investigate the underlying structure of two different sensitive kernels, resulting from possible formulations connecting sound velocity perturbations $(\delta c)$ with travel-times variations of first arrivals $(\delta t)$. These operators are central to several inversion techniques, thus justifying this particular interest for the development of better strategies for water layer velocity reconstruction departed from OBN data. Both cases considered herein assume linearization about a reference-state profile, which is often justified by small sound velocity perturbations associated with typical termohaline variations (MUNK and WUNSCH, 1979; MUNK, 1986). Additional assumption of modal-shape aspect for the velocity perturbations further reduces the parameter space, and allows a more direct inspection of resultant linear operators.

\section{Ocean data $\left(c_{0}\right.$ and $\left.\delta c\right)$}

In the absence of direct in situ measurements of ocean properties, one possible by-pass is to make direct use of freely-available Ocean Reanalysis data (SOUZA et al., 2021). These models combine historical ocean observations with a general ocean model (typically computational) driven by historical estimates of surface winds, heat, etc, combined with data assimilation to reconstruct historical changes in the state of the ocean. Figure 1 exemplify a (monthly) temporal variation of a 1D water sound velocity profile, $c(z, t)$, that can be computed with reanalysis products and the Thermodynamic Equation Of Seawater - 2010. From these, a direct estimate of time-averaged water profile $c_{0}(z)$ is readily obtained, along with Hovmöller diagrams showing $\delta c(z, t)$ spatio-temporal varying behavior. 

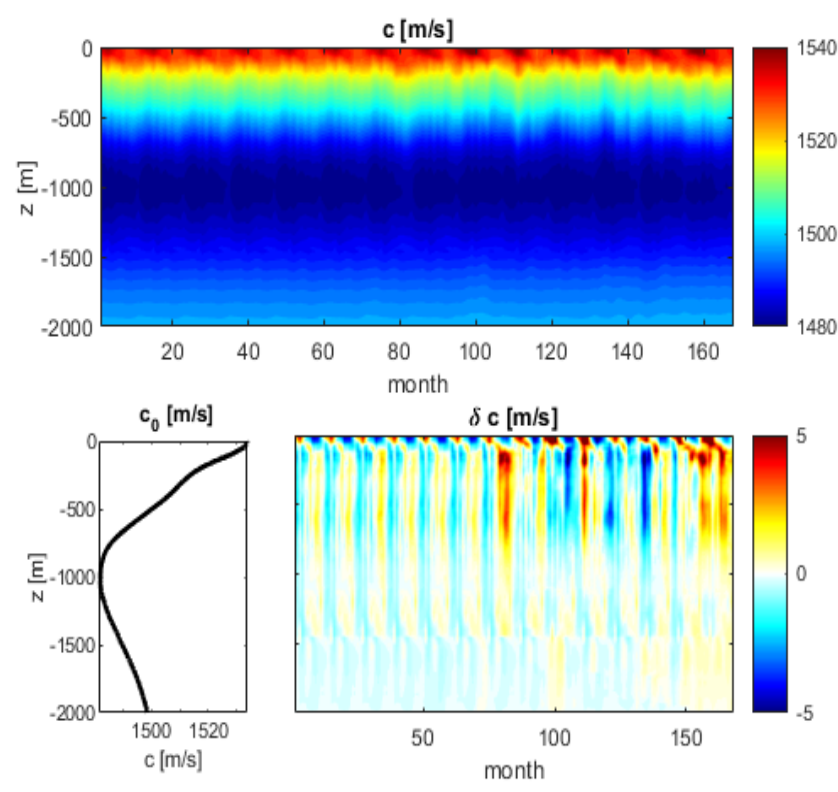

Figure 1: (top) Historical evolution of a 1D water sound velocity profile, (lower-left) time-averaged profile, (lowerright) Hovmöller diagram of $\delta c$ variations.

Statistically persistent patterns perceived in fluctuating quantities can usually be associated with some intrinsic variability of coherent spatio-temporal structures that, for instance, may play a critical role in seasonal forecasting of global-scale weather phenomena (BIERKENS and Van BEEK, 2009). The most common method adopted within the weather and climate community to identify coherent structures is called empirical orthogonal function (EOF) analysis (HANNACHI et al., 2007).

Standard EOF analysis, also known as proper orthogonal decomposition (POD), principle component analysis (PCA), among some other names, is a modal decomposition technique that extracts coherent structures, or patterns, in the form of modes of variability from data. These modes are computed as the eigenvectors of the (spatially weighted) anomaly covariance matrix. The corresponding eigenvalues measure the variance and optimally rank the underlying modes within the fluctuating pattern. In the past few decades, traditional and more advanced variants of this technique have been used to study the modes of variability/predictability of fluid flows and the Earth system (TAIRA et al., 2017).

In Figure 2, we exemplify some possible modedecomposition applied to $\delta c(z, t)$ temporal evolution, shown previously in from Figure 1. The first case stands for traditional PCA, where we highlight (in red) the first three optimally ranked modal decomposition, following by their spatio-temporal characteristics. A second result is related to Dynamic Mode Decomposition (DMD), where some temporal behavior is enforced in the spatial patterns (SCHMID et al., 2011). Again, three underlying (non-normal) modal representations of $\delta c(z, t)$ are selected, as Fourier decomposition is imposed on the temporal variation. At last, we display enforced Fourier decomposition along the space direction, aiming for more controlled representation of $\delta c(z, t)$, regardless of their time evolution. Here a pair of cossine/sine wavelengths ( $\pi$ and $8 \pi$ ) of a Fourier series, having exponential decay with increasing depth, are shown to exemplify this possible choice of (multi-scaled) orthogonal basis emphasizing the uppermost part of the ocean variation. Without lost of generality, we carry our analysis based on this last option, which bears the fundamental idea behind a further reduction in the parameter space in the velocity model, by some form of mode decomposition of $\delta c(z, t)$. The choice of 4 parameters considerably reduces the linear operator, and allows direct evaluations of the null-space behavior.

\section{Two Sensitivity Kernels}

The study on how travel/arrival times are affected by the sound-speed distribution in the water, and how the latter can be obtained by inversion, was first introduced by MUNK and WUNSCH (1979), as a remote-sensing technique (Ocean Acoustic Tomography) for large-scale monitoring of the ocean interior using low-frequency sound. Despite being a high-frequency asymptotic approximation, raytheoretic modeling has been the most common approach in this field, with variations of the sound speed along the geometric ray paths providing a kernel where inversion of travel time perturbation can be converted to ocean perturbation. As an alternative to some limitations of ray-theoretic modeling, wave-theoretic approaches have also been proposed, such as modal arrival (MUNK and WUNSCH, 1979; SHANG, 1989) and peak arrival approaches (SKARSOULIS and CORNUELLE, 2004; SARKAR et al., 2011).

Regardless of one personal choice of propagation modeling, the vertically distributed sources separated over thousands of kilometers, promote a very distinct situation for the transmitted arrivals that are to be expected in horizontally distributed OBN-type aquisitions. Since variations of the velocity are fairly small, for instance, simulated raypaths can be assumed to be straight (or very nearly so) for some near-offset region (BERTRAND and MACBETH, 2003). On the other hand, wave-theoretic formulation could be more sensitive to the low-frequency small velocity variations, leading to better inversion results. In this study, we choose to investigate both.

\section{Straigh Ray Sensitivity Kernel (SRSK)}

In ray-theory, the linearization of the classic relationship of perturbed travel-times $(\delta t)$ with the varying velocity $(\delta c)$ is given by

$$
\delta t=t-\int_{s} \frac{1}{c_{0}(z)} d \vec{s} \approx-\int_{s} \frac{\delta c(z)}{c_{0}(z)^{2}} d \vec{s},
$$

where $\vec{s}$ stands for the ray-path. Here we assume that $c_{0}(z)$ and $\delta c(z)$ are predominantly depth-dependent functions, at least locally for each shot. For straight ray approximation, parametrization of line segments allows this integral to be rewritten as

$$
\delta t \approx-\int_{0}^{1} \frac{\delta c(\phi)}{c_{0}(\phi)^{2}}\left|\phi^{\prime}\right| d \phi,
$$

where $\phi$ is a controlling parameter for the current position along the line segment, and $\left|\phi^{\prime}\right|=\sqrt{\phi_{x}^{2}+\phi_{z}^{2}}$ can be seen as a scale factor associated with the ray inclination. 

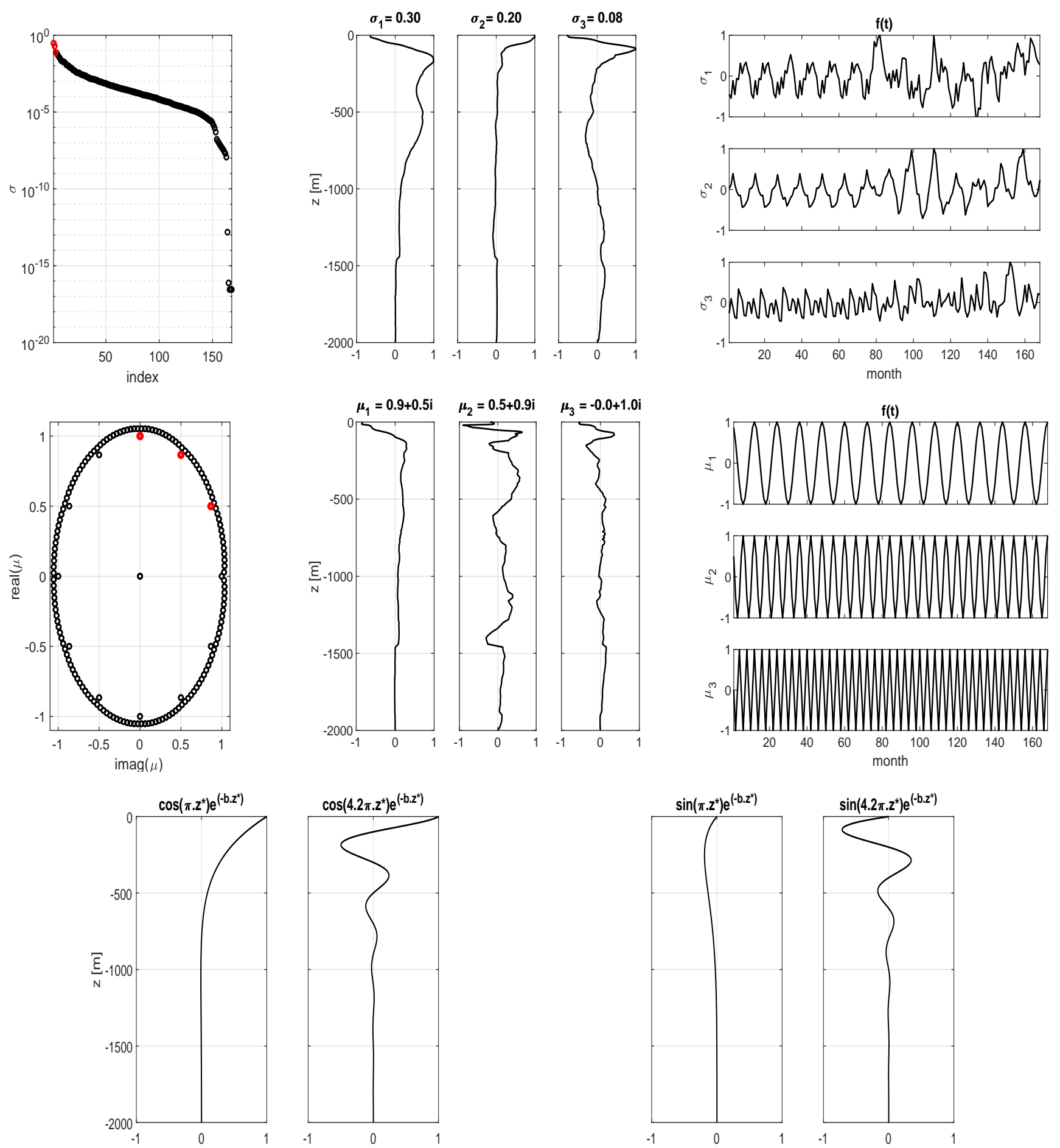

Figure 2: Some possible mode decompositions for $\delta c(z, t)$. (top row) PCA results, first displaying the correspondent singular values $(\sigma)$, and the spatio and temporal behavior of first three optimally ranked modes (red). (middle row) DMD results, showing the spectral behavior of obtained eigensolutions, followerd by spatio-temporal behavior of three highligthed modes (red). (bottom row) Fourier series decomposition of the spatial pattern of $\delta c(z, t)$, having (left) varying cossine terms with decay along normalized depth $\left(z^{*}\right)$, and (right) the sinusoidal correspondents. The decay rate is here given by $b=8$. 


\section{Pressure Sensitivity Kernel (PSK)}

The more elaborated connection with the perturbation form of the Helmholtz equation and the transmitted arrivals, have already been described in more detail elsewhere (SKARSOULIS and CORNUELLE, 2004; SARKAR et al., 2011; WU et al., 2020). The fundamental idea here is that a perturbation $\delta c$ in the sound speed reference state can be associated to $\delta G$ perturbations of the Green's function, through the first Born approximation. These perturbations, in turn, can also be related to $\delta p$ variations of the acoustic pressure perceived at the receiver in the time domain. The connection with $\delta t$ can be established by the application of a signal envelope on the complex $\delta p$, forming an arrival pattern that allows one to identify peaks arrivals (SKARSOULIS and CORNUELLE, 2004). For the wave-type Helmholtz equation, a $\delta t$ perturbation is related to the underlying $\delta c$ velocity variation by,

$$
\delta t=\int_{V} K\left(\mathbf{r}^{\mathbf{9}} \mid \mathbf{r}_{s} ; \mathbf{r}_{r} ; c_{0}\right) \delta c\left(\mathbf{r}^{\mathbf{\prime}}\right) d V\left(\mathbf{r}^{\mathbf{9}}\right),
$$

where $K\left(\mathbf{r}^{\prime} \mid \mathbf{r}_{s} ; \mathbf{r}_{r} ; c_{0}\right)$ is known as the integration kernel. This kernel accounts to the spatial sensitivity of each point in a considered domain, for a fixed source $\left(\mathbf{r}_{s}\right)$ and receiver $\left(\mathbf{r}_{r}\right)$ pair, having also some a priori knowledge of a base-state velocity profile $c_{0}(\mathbf{r})$ and the underlying features of its acoustic response $\left(\tau_{0}\right)$. For a finitefrequency wave-theoretic modeling, $K\left(\mathbf{r}^{\prime} \mid \mathbf{r}_{s} ; \mathbf{r}_{r} ; c_{0}\right)$ is given as (SKARSOULIS and CORNUELLE, 2004)

$$
\begin{aligned}
K\left(\mathbf{r}^{\prime} \mid \mathbf{r}_{s} ; \mathbf{r}_{r} ; c_{0}\right)=\mathscr{R} & \left\{\frac{v_{0}-i w_{0}}{2 \pi b} \int_{-\infty}^{\infty} i \omega Q\left(\mathbf{r}^{\prime} \mid \mathbf{r}_{s} ; \mathbf{r}_{r} ; \omega ; c\right) e^{i \omega \tau_{0}} d \omega\right. \\
& \left.+\frac{\dot{v}_{0}-i \dot{w}_{0}}{2 \pi b} \int_{-\infty}^{\infty} Q\left(\mathbf{r}^{\prime} \mid \mathbf{r}_{s} ; \mathbf{r}_{r} ; \omega ; c\right) e^{i \omega \tau_{0}} d \omega\right\}
\end{aligned}
$$

Quantities $v_{0}, w_{0}, \dot{v}_{0}, \dot{w}_{0}$ (overdot denotes differentiation with respect to time) and $b$ are related to the notion of small deviations in the pressure amplitude pattern, i. e. assuming minor variations of the signal envelope-shape about a known peak arrival $\tau_{0}$, evaluated at the background (unperturbed) velocity base-sate $c_{0}(\mathbf{r})$. Expressing the complex pressure $p(t, c)$, in terms of its real and imaginary parts, $p(t, c)=v(t, c)+i w(t, c)$, the signal envelope is then given by its absolute quantity $|p(t, c)|$. Peak arrivals are here defined as the significant local maxima, $\partial|p(t, c)| / \partial t=$ 0 , of the arrival envelope pattern. Minor deviations $\delta t$ of such peaks can be expressed by a first-order Taylor expansion, which then gives $b=\dot{v}_{0}^{2}+v_{0} \ddot{v}_{0}+\dot{w}_{0}^{2}+w_{0} \ddot{w}_{0}$. All quantities here are evaluated directly from data, once the expected arrivals of known sound velocity base-state are known. More details can be found on related references (SKARSOULIS and CORNUELLE, 2004).

Thus, the main part of this sensitivity kernel is actually $Q\left(\mathbf{r}^{\prime} \mid \mathbf{r}_{s} ; \mathbf{r}_{r} ; \omega ; c\right)=\partial\left(G\left(\mathbf{r}_{r}, \mathbf{r}_{s}\right)\right) / \partial c\left(\mathbf{r}^{\prime}\right)$, which relates the Green's function for source and receiver, at certain angular frequency $(\omega)$, with the source-signal spectrum $\left(P_{S}\right)$, as

$$
\begin{array}{r}
Q\left(\mathbf{r}^{\prime} \mid \mathbf{r}_{s} ; \mathbf{r}_{r} ; \omega ; c\right)=G\left(\mathbf{r}^{\prime} \mid \mathbf{r}_{s} ; \omega ; c\right) \\
G\left(\mathbf{r}_{r} \mid \mathbf{r}^{\prime} ; \omega ; c\right) \\
\times \frac{2 \omega^{2} P_{s}(\omega)}{c^{3}\left(\mathbf{r}^{\prime}\right)} .
\end{array}
$$

By the inverse Fourier transform of this inner structure, we reach what is known as the Pressure Sensitivy Kernel (PSK) (SARKAR et al., 2011), that will be our focus here.

In a horizontally stratified (range-independent) background marine environment, normal-mode theory offers a possibility to obtain the background Green's function $G$. For an axisymmetric point source representation about the vertical axis, a three-dimensional cylindrical coordinate system $(r, z, \theta)$ is used with its origin at the sea surface and the source located on the vertical $z$ axis (positive downward) at $z=z_{s}$. In this situation, the background sound speed is only depth-dependent $c(z)$. A far-field approximation (of the outgoing Hankel dependency) for the Green's function at $(r, z)$ is then given by (JENSEN et al., 2011),

$$
G\left(r, z \mid z_{s} ; \omega ; c\right)=\frac{e^{-i \pi / 4}}{\rho \sqrt{8 \pi}} \sum_{n=1}^{M} \frac{u_{n}\left(z_{s}\right) u_{n}(z)}{\sqrt{k_{n} r}} e^{i k_{n} r},
$$

where $\rho$ is the water density and $k_{n}$ and $u_{n}, n=1, \ldots, M$, are the real eigenvalues and corresponding eigenfunctions (propagating modes) of a vertical Sturm-Liouville problem, suplemented by pressure-release surface $\left(u_{n}(0)=0\right)$ and a perfectly rigid bottom $\left(\partial u_{n} /\left.\partial z\right|_{z=H}=0\right)$ boundary conditions. The characteristics of such eigenvalue problem are vastly well-known (JENSEN et al., 2011). Figure 3 exemplify some of the obtained propagating modal solutions for the time-averaged reference profile. These are plugged back into eq. (6) and (5) to form the dot product between source and receiver Green's function, for each frequency. Finally, the time contribution is obtained by Fourier synthesis, eq. (4), through combination o several frequency-dependent Green's-products at some known arrival time $\tau_{0}$.
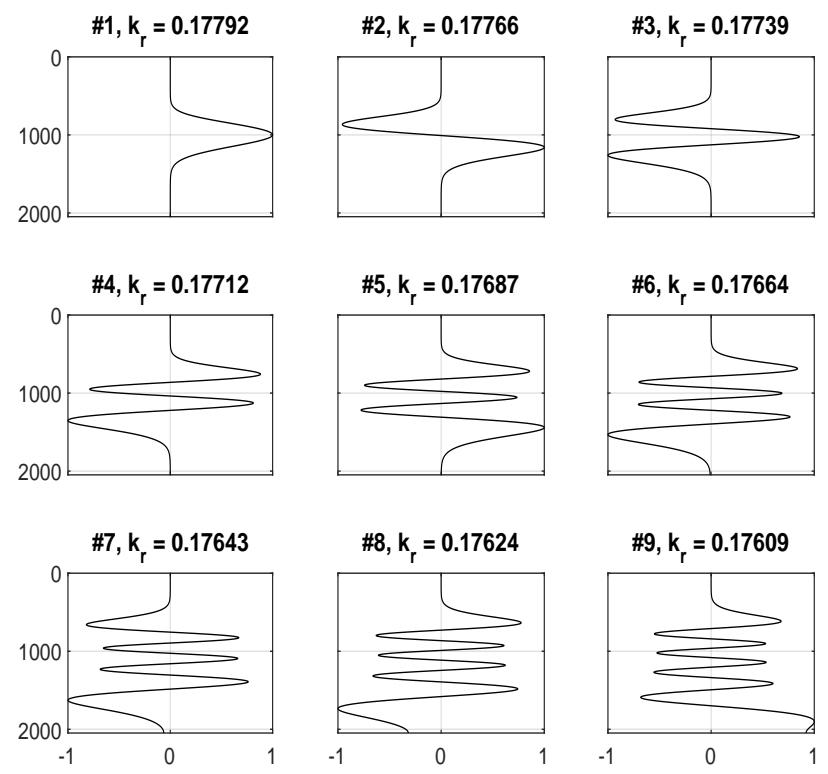

Figure 3: Sample of real (propagating) eigenfunctions obtained for the time-averaged velocity profile at $42 \mathrm{~Hz}$.

\section{Results}

For the evaluation of sensitivity kernels, we consider a single shot being received at 5 bottom-node offset locations 
(500 m, $1500 \mathrm{~m}, 2500 \mathrm{~m}, 4000 \mathrm{~m}, 6000 \mathrm{~m})$, having $c_{0}$ as the background time-averaged velocity profile (Figure 1). Each source/receiver pair composes a row in these sensitivity matrices, whereas each column are associated with modal-coefficients $\left(a_{i}\right)$ of the parameterized form imposed in $\delta c(z)$. Since modal decomposition allow us to define

$$
\delta c(z)=\sum_{i}^{4} a_{i} F_{i}(z),
$$

each matrix term corresponds to a integration being performed in eq. (2) and eq. (4), that is weighted by the $F_{i}(z)$ decaying Fourier series patterns. Figure 4 presents the obtained singular values for the two formulated sensitivity kernels. For the PSK calculations, the source spectrum $P(\omega)$ is considered as a Gaussian profile, centered at $45 \mathrm{~Hz}$ with $5 \mathrm{~Hz}$ of width. The frequency integration is carried over $42 \mathrm{~Hz}$ up to $48 \mathrm{~Hz}$, with steps of $0.05 \mathrm{~Hz}$. Uniformly distributed grid, with $3 \mathrm{~m}$ of resolution, is enforced in the spatial discretization.

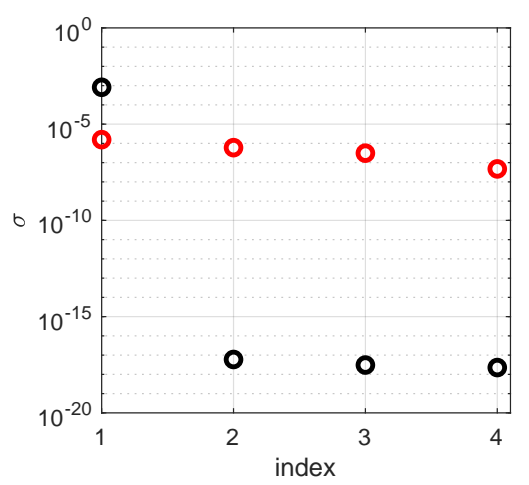

Figure 4: Obtained singular values of the resultant linear operators related to (black) SRSK and (red) PSK.

Here we are able to confirm the limitations related to the use of "vertically" straight-ray approximation with a horizontally varying perturbations, as only one singular value is verified (VESNAVER, 1995). Conversely, wavetheoretic provides a broader row-space for coefficient determination in the inversion. Figure 5 displays a more detailed view for the reasons why PSK structure provide a reduction of the null-space. Not only different offsets promote changes in banana-doughnut first Fresnel zones, but also the upper part becomes gradually disturbed (to the right) resulting in a misalignment from the near-offset results.

\section{Conclusion}

In this study we took a closer look on the sensitivity kernel structure associated with horizontally distributed acquisition arrays, considering the use modal decompositions of the water properties applied in two possible formulations for the transmitted waves reaching the ocean bottom receptors. The results suggests that wave-theoretic sensitive kernels displays a great reduction in the null-space, thus being able to update more coefficients per iteration unambiguously without introducing additional assumptions in some inversion
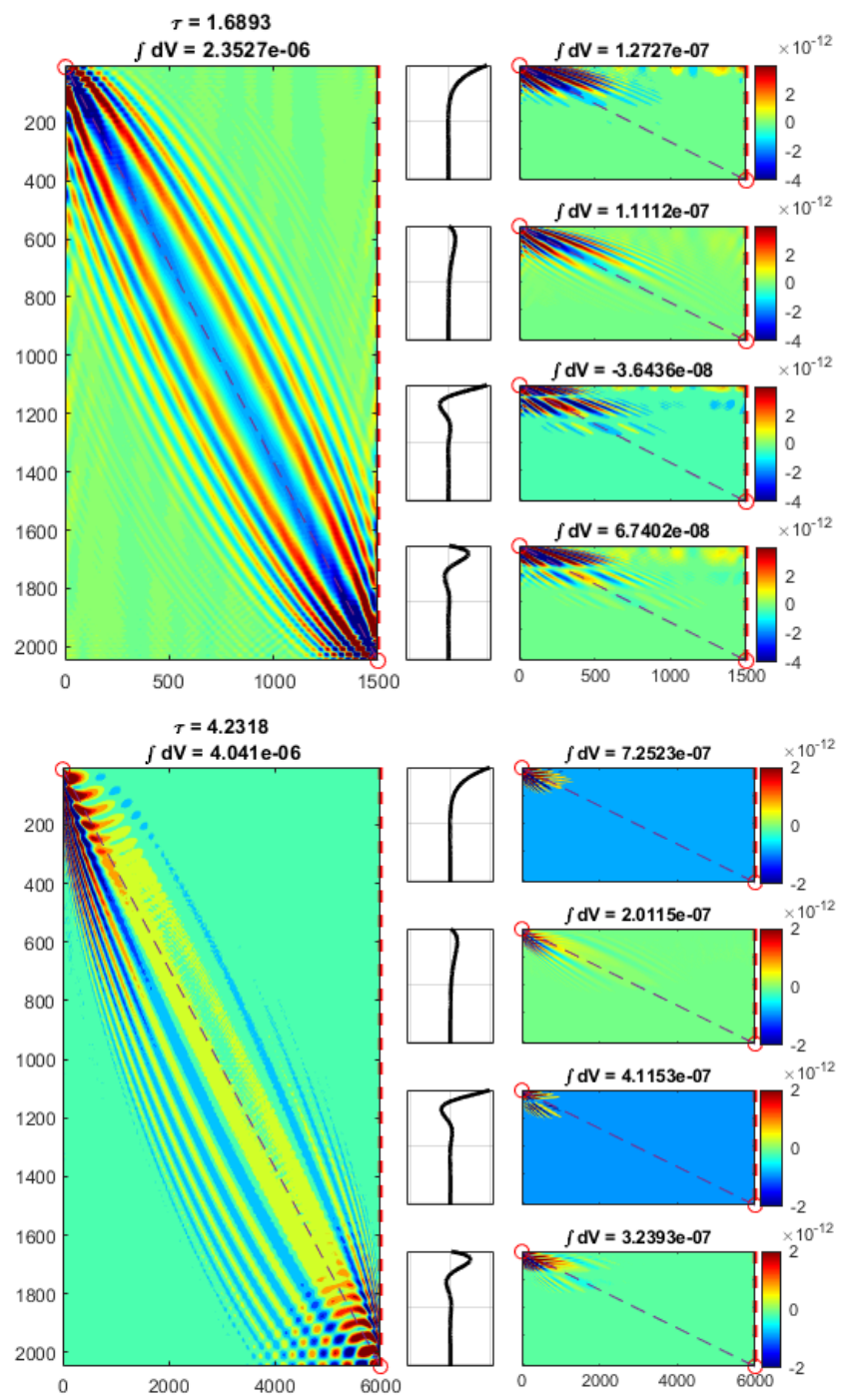

Figure 5: Obtained singular values of the resultant linear operator related to the of (propagating) eigenfunctions obtained for the time-averaged velocity profile at $42 \mathrm{~Hz}$.

scheme, when compared to the straight ray approach. Although this finding may lead one to readily discard the straight-ray approach, we found that it might still be some ways to explore it. A current idea accounts some spatial multi-scale iterative scheme to update subsequent travel-time residuals, that are to be tested in the future, but were initiated in a companion paper (PAIVA et al., 2021).

\section{Acknowledgements}

The authors are grateful to Pedro Lins de Souza for providing the reanalysis data, and to André Bulcão and Bruno Dias for the fruitful backstage discussions during a monography defense. This research was supported by Petrobras through "Determinação da velocidade do som da coluna d'àgua utilizando dados de levantamentos sísmicos" project with Universidade Federal of Santa Catarina (UFSC), and ANP through the R\&D levy regulation, process: 2017/00071-6. 


\section{References}

AHMED, I.; ROBERTS, M., and NGUYEN, B., 2013, Joint inversion for ocean bottom node position and average water velocity along the shot line, in SEG Technical Program Expanded Abstracts 2013, 4880-4884, Society of Exploration Geophysicists.

AMINI, A.; PENG, H.; ZHANG, Z.; HUANG, R., and YANG, J., 2016, Joint inversion of water velocity and node position for ocean-bottom node data, in SEG Technical Program Expanded Abstracts 2016, 5490-5494, Society of Exploration Geophysicists.

AYOBAMI, A.; HEPP, D. A., and TOBIAS, M., 2020, Correction of water column height variation on $2 \mathrm{~d}$ grid high-resolution seismic data using dgps based methodology: Scientific Reports (Nature Publisher Group), 10.

BERTRAND, A. and MACBETH, C., 2003, Seawater velocity variations and real-time reservoir monitoring: The Leading Edge, 22, 351-355.

BERTRAND, A.; RIBEIRO, C., and MACBETH, C., 2004, Uncertainties in the $4 d$ signature due to seawater velocity variations: Society of Exploration Geophysicists.

BIERKENS, M. and Van BEEK, L., 2009, Seasonal predictability of european discharge: Nao and hydrological response time: Journal of Hydrometeorology, 10, 953-968.

BOELLE, J.-L.; LECERF, D.; LAFRAM, A., and CANTILLO, J., 2010, Ocean bottom node processing in deep offshore environment for reservoir monitoring, in SEG Technical Program Expanded Abstracts 2010, 41904194, Society of Exploration Geophysicists.

BRIGHT, D.; JONES, C. E., and SELVAGE, J. I., 2015, Solving water column statics with seismic oceanography: 77th EAGE Conference and Exhibition 2015, 1-5.

DOCHERTY, P. and HAYS, D., 2012, Ambiguities in direct arrival time inversion for ocean bottom nodes: 74th EAGE Conference and Exhibition incorporating EUROPEC 2012, cp-293.

DUNN, R. A., 2015, Ocean acoustic reverberation tomography: The Journal of the Acoustical Society of America, 138, 3458-3469.

GUERRA, C.; CARBONESI, J., and RITTER, G., 2015, Dynamic correction for water-velocity and tidal variations: 14th International Congress of the Brazilian Geophysical Society \& EXPOGEF, Rio de Janeiro, Brazil, 3-6 August 2015, 1351-1354.

HANNACHI, A.; JOLLIFFE, I., and STEPHENSON, D., 2007, Empirical orthogonal functions and related techniques in atmospheric science: A review: International Journal of Climatology: A Journal of the Royal Meteorological Society, 27, 1119-1152.

HOLBROOK, W. S.; PÁRAMO, P.; PEARSE, S., and SCHMITT, R. W., 2003, Thermohaline fine structure in an oceanographic front from seismic reflection profiling: Science, 301, 821-824.

JENSEN, F. B.; KUPERMAN, W. A.; PORTER, M. B., and SCHMIDT, H., 2011, Computational ocean acoustics: Springer Science \& Business Media.

LACOMBE, C.; BUTT, S.; MACKENZIE, G.; SCHONS, M., and BORNARD, R., 2009, Correcting for water-column variations: The Leading Edge, 28, 198-201.

MUNK, W., 1986, Acoustic monitoring of ocean gyres: Journal of Fluid Mechanics, 173, 43-53.
MUNK, W. and WUNSCH, C., 1979, Ocean acoustic tomography: A scheme for large scale monitoring: Deep Sea Research Part A. Oceanographic Research Papers, 26, 123-161.

PAIVA, L.; LAJÚS, F. Jr., and KLEIN, A., 2021, Parameterized water layer reconstruction for first arrivals in obn data: Presented at the 17th International Congress of Brazilian Geophysical Society \& EXPOGEF, Rio de Janeiro, Brazil, 8-11 November 2021.

RITTER, G. L. S., 2010, Water velocity estimation using inversion methods: Geophysics, 75, U1-U8.

SARKAR, J.; CORNUELLE, B. D., and KUPERMAN, W., 2011, Information and linearity of time-domain complex demodulated amplitude and phase data in shallow water: The Journal of the Acoustical Society of America, 130, 1242-1252.

SCHMID, P.; LI, L.; JUNIPER, M., and PUST, O., 2011, Applications of the dynamic mode decomposition: Theoretical and Computational Fluid Dynamics, 25, 249-259.

SHANG, E., 1989, Ocean acoustic tomography based on adiabatic mode theory: The Journal of the Acoustical Society of America, 85, 1531-1537.

SHEEN, K., 2019, New wave seismics: 81st EAGE Conference and Exhibition 2019 Workshop Programme, $1-2$.

SKARSOULIS, E. K. and CORNUELLE, B. D., 2004 , Travel-time sensitivity kernels in ocean acoustic tomography: The Journal of the Acoustical Society of America, 116, 227-238.

SONG, H.; PINHEIRO, L. M.; RUDDICK, B., and HUANG, $X ., 2012$, Seismic oceanography: a new geophysical tool to investigate the thermohaline structure of the oceans, in Oceanography, 113-128, InTech Press.

SOUZA, J.; COUTO, P.; SOUTELINO, R., and ROUGHAN, M., 2021, Evaluation of four global ocean reanalysis products for new zealand waters-a guide for regional ocean modelling: New Zealand Journal of Marine and Freshwater Research, 55, 132-155.

TAIRA, K.; BRUNTON, S. L.; DAWSON, S. T.; ROWLEY, C. W.; COLONIUS, T.; MCKEON, B. J.; SCHMIDT, O. T.; GORDEYEV, S.; THEOFILIS, V., and UKEILEY, L. S., 2017, Modal analysis of fluid flows: An overview: Aiaa Journal, 55, 4013-4041.

UDENGAARD, C. and CRAFT, K., 2012, Analysis of water column complexity in obn data: 74th EAGE Conference and Exhibition incorporating EUROPEC 2012, cp-293.

VESNAVER, A., 1995, Null space reduction in the linearized tomographic inversion, in Full Field Inversion Methods in Ocean and Seismo-Acoustics, 139-145, Springer.

WOMBELL, R., 1996, Water velocity variations in 3d seismic processing, in SEG Technical Program Expanded Abstracts 1996, 1666-1669, Society of Exploration Geophysicists.

WU, W.; ZHAN, Z.; PENG, S.; NI, S., and CALLIES, J., 2020, Seismic ocean thermometry: Science, 369, 15101515.

ZOU, Z.; ZHOU, H.-W.; GURROLA, H.; BIAN, A.; HUANG, Z., and ZHANG, J., 2018, Impact and solutions of seawater heterogeneity on wide-angle tomographic inversion of crustal velocities in deep marine environments-numerical studies: Journal of Earth Science, 29, 1380-1389. 